\section{Educação para a saúde em osteoporose com idosos de um programa universitário: repercussões}

\author{
Heath education on osteoporosis \\ for elderly university students
}

\author{
${ }^{1}$ Departamento de Nutrição, \\ Universidade Federal \\ do Piauí, Teresina, Brasil. \\ 2 Departamento de Medicina \\ Comunitária, Universidade \\ Federal do Piaui, \\ Teresina, Brasil. \\ Correspondência \\ Cecília Maria Resende \\ Gonçalves de Carvalho \\ Campus Universitário \\ Petrônio Portela, \\ SG-13, Teresina, $P I$ \\ 64049-550, Brasil. \\ cecilia@webone.com.br
}

\begin{abstract}
The purpose of this study was to analyze knowledge and concepts in relation to osteoporosis and changes in eating habits among elderly university students before and after an educational intervention. An action-based research strategy applied questionnaires to 95 individuals ranging in age from 60 to 86 years, with or without osteoporosis. The work showed an obvious lack of knowledge concerning important information about the disease and measures needed to better control progressive loss of bone mass. After four months of educational activities, more than half of the group had reported dietary changes. We emphasize the need to adopt educational strategies not only to inform on ideal preventive practices for osteoporosis but also to build a new mindset and behavior, which are important to control this condition.
\end{abstract}

Osteoporosis; Aging; Health Education; Food Habits; Health Promotion
Cecília Maria Resende Gonçalves de Carvalho 1 Carla Cristina Carvalho Fonseca 1 José Ivo Pedrosa 2

\section{Introdução}

A osteoporose é uma doença sistêmica que resulta em reduzida massa óssea e deterioração da micro-arquitetura do tecido ósseo, levando à fragilidade mecânica e conseqüente predisposição a fraturas com trauma mínimo, atingindo a todos, em especial a mulheres após a menopausa.

A doença é considerada uma importante questão de saúde pública mundial devido a sua alta prevalência, em função dos seus efeitos devastadores na saúde física e psicossocial, com grandes prejuízos financeiros. Causa invalidez pelas deformidades e incapacidades dos indivíduos afetados e, pelo demorado tratamento das fraturas decorrentes da enfermidade, gera um ônus elevado. As fraturas de quadril reduzem o tempo de vida em $36 \%$ para homens e $21 \%$ para mulheres 1 , ocorrendo a morte nos primeiros seis meses depois da fratura de colo do fêmur. Em pacientes com desordens psiquiátricas, a taxa de mortalidade chega a $50 \%$ após a fratura 2 .

O custo anual com o tratamento de fraturas osteoporóticas, em 1995, segundo o National Osteoporosis Foundation, chegou a 13,8 bilhões de dólares, uma quantidade que pode superar o dobro nos próximos 25 anos por causa do crescimento da população idosa 3 .

No Brasil, a população propensa a desenvolver osteoporose aumentou de 7,5 milhões 
em 1980 para 15 milhões no ano 2000 4, chegando a acometer $35 \%$ a $52 \%$ das mulheres com mais de cinqüenta anos e uma proporção de $19 \%$ a $39 \%$ dos homens. Vinte entre cada cem mulheres são portadoras de doenças osteoporóticas, com 4 milhões e 400 mil casos, e um gasto de mais de 1 bilhão e 300 milhões de reais/ano 5 .

A etiologia da perda de massa óssea é complexa, multifatorial, inclui hereditariedade, etnia, idade avançada, sexo feminino, baixo peso corporal, deficiência hormonal, excessivo consumo de álcool, inatividade física, tabagismo, e fatores nutricionais.

Embora já estejam bem estabelecidos os benefícios das mudanças nos hábitos de vida como um importante fator modificável relacionado à saúde óssea, a sua importância e o conhecimento de que a prevenção de perda de massa óssea pode ser feita com alimentação bem balanceada e a prática regular de exercício físico nem sempre é do conhecimento da população.

Para a promoção de saúde educar é contribuir para a autonomia das pessoas, é considerar a afetividade, a amorosidade, a capacidade criadora e a busca da felicidade como igualmente relevantes e como indissociáveis das demais dimensões da vida humana e, portanto, promover a saúde não pode ser uma ação descolada dos sentidos atribuídos à vida que se somam aos saberes acumulados tanto pelas ciências quanto pelas tradições culturais locais e universais 6 .

Alguns programas educativos têm surgido no sentido de orientar os participantes em nível de prevenção e auxiliá-los no tratamento da osteoporose. Com essa perspectiva, Sedlak et al. 7 desenvolveram programas educativos dirigidos aos indivíduos e familiares com ênfase à prevenção da osteoporose nos níveis primário, secundário e terciário. Embora todos os participantes do programa tivessem um maior nível de conhecimento da doença, após três semanas os mesmos não adotaram comportamentos mais preventivos.

Já o estudo desenvolvido por Satterfield et al. 8 , sobre a percepção das mulheres idosas americanas em relação aos fatores de risco da osteoporose e do tratamento da doença, demonstrou que os itens dieta e exercício foram aqueles que alcançaram o mais alto escore de aprendizado. Quanto mais o grupo entendia o papel da dieta e do exercício em relação à doença mais apresentava melhoria nas mudanças de comportamento.

Para avaliar se a educação em osteoporose contribui com mudanças no estilo de vida e no comportamento das pessoas em relação ao tratamento terapêutico, Roinick et al. 9 investigaram 508 mulheres, com idade entre 54 a 65 anos, com ou sem osteoporose. Após seis meses, mais da metade do grupo que recebeu atividades educativas reportaram mudanças na dieta $(\mathrm{p}<0,001)$ e no consumo de cálcio $(\mathrm{p}<0,01)$, enquanto $43 \%$ aumentaram seu consumo de vitamina $\mathrm{D}(\mathrm{p}<0,0001)$ quando comparados às mulheres do grupo não exposto às atividades de educação. A melhoria no ganho da massa óssea observada pelo exame de densitometria óssea foi associada ao início da terapia de reposição hormonal (9\%) e uso do Alendronato (5\%), além do aumento no consumo de vitamina $\mathrm{D}$.

No Brasil, a adoção de uma pedagogia para o envelhecimento bem sucedido começou a partir década de 1980 nas universidades. Consideradas como instituições de grande alcance social, as universidades da terceira idade desempenham um importante papel na manutenção da qualidade de vida dos indivíduos envelhecentes. As experiências das Universidades Abertas da Terceira Idade são importantes e devem estar vinculadas, principalmente, à formação de recursos humanos, à pesquisa e à integração de profissionais nas questões relativas à velhice e ao envelhecimento 10

As adoções de práticas educativas para prevenção e tratamento da osteoporose, no Brasil, têm sido preconizadas pela fisiatra Pérola Grienberg Plaper 11, através da criação da "Escola de Osteoporose" que está conseguindo modificar hábitos e reabilitar os pacientes que ali freqüentam.

O interesse na realização do estudo é contribuir para tornar o idoso mais informado e consciente dos problemas relacionados com a osteoporose, além de torná-los agentes sociais multiplicadores desse conhecimento. Nesse sentido, com os idosos que participam do Programa Terceira Idade em Ação (PTIA), da Universidade Federal do Piauí (UFPI), foram realizadas entrevistas com o objetivo de conhecer o significado que os idosos matriculados no PTIA atribuem à osteoporose e suas implicações, identificar concepções, hábitos e atitudes em relação à doença e à alimentação, antes e após a aplicação de um programa de intervenção educativa.

\section{Casuística e métodos}

\section{Caracterização do universo}

Em 1998, a UFPI, criou o PTIA como um curso de extensão com duração de dois anos, oferecendo vagas para as pessoas com sessenta anos 
ou mais, com o objetivo de compreender e informar à comunidade piauiense sobre a realidade do idoso e formar esses cidadãos para que sejam capazes de criar mecanismos que levem a ações que contribuam para a melhoria das condições de vida do indivíduo no período do envelhecimento. O programa oferece, atualmente, um elenco de 25 disciplinas em diversas áreas do conhecimento, além de outras atividades culturais e de lazer, voltadas especificamente para esse grupo etário.

Dos participantes do curso $41,7 \%$ eram casados e viviam com o cônjuge, $37,5 \%$ eram viúvos e $20,8 \%$ eram solteiros. Muitos eram proprietários dos imóveis em que residiam e 90\% moravam em casas e $10 \%$ em apartamentos. O sexo feminino é o predominante, confirmando as tendências de maior esperança de vida ao nascer para as mulheres 12 .

A escolaridade dos idosos é muito variável, cerca de $63,5 \%$ têm o segundo grau completo, $20 \%$ não concluíram o primeiro grau, $12,5 \%$ concluíram o primeiro grau e $5 \%$ têm o curso superior. O perfil etário compreendeu uma população de idosos na faixa de 60 a 86 anos, com ou sem osteoporose.

\section{Características do estudo}

O modelo do estudo é da pesquisa-ação inspirada na sua concepção original de educação pela prática onde se espera modificar determinado comportamento dos sujeitos envolvidos pela intervenção ativa de atores - os pesquisadores 13 .

A pesquisa foi desenvolvida entre março e dezembro de 2001, em três etapas. Na primeira delas se fez o levantamento das condições sócio-econômicas, morbidade referida, resultados de densitometria óssea, quedas, alimentação, atividade física, variável comportamental relativa a hábitos alimentares como a ingestão de cafeína, uso de cigarros, bebidas alcoólicas e consumo alimentar por meio da aplicação de um questionário padronizado e previamente testado, que também continha questões relativas ao conhecimento sobre a doença, fatores de risco relacionados e medidas de prevenção. $\mathrm{Na}$ segunda etapa, foram desenvolvidas as ações educativas e a terceira foi dedicada à obtenção de dados pós-intervenção, através da aplicação de outro questionário para avaliar o resultado da intervenção educativa na aquisição de conhecimentos e atitudes dos sujeitos e avaliar a qualidade das aulas ministradas. Neste segundo instrumento, repetiu-se a questão relativa à variável comportamental e de informações sobre a doença, fatores de risco e me- didas de prevenção, temas que foram abordados nas práticas educativas.

Dentre os fatores que predispõem ao desenvolvimento da osteoporose foram trabalhados nas ações educativas, especialmente, a doença, prevenção de quedas, sedentarismo, tabagismo, consumo de bebidas alcoólicas, refrigerantes e de cafeína, ingestão alimentar de cálcio e vitamina $\mathrm{D}$.

\section{Os participantes e a dinâmica da pesquisa}

A disciplina de osteoporose foi oferecida aos idosos matriculados no PTIA, em três turmas com cerca de 33 alunos em cada uma delas.

Para todos que efetivamente cursaram a disciplina "osteoporose" $(n=95)$ foi entregue o questionário e dadas as orientações para o preenchimento correto. O questionário continha questões de múltiplas escolhas e questões abertas sobre o significado e conhecimento dos sujeitos sobre osteoporose, alimentação e atividade física.

A identificação dos indivíduos com osteoporose ocorreu a partir da informação de cada entrevistado, tendo-se o cuidado de confirmar as informações pelo exame de densitometria óssea já realizados.

A intervenção educativa significa o conjunto de atividades desenvolvidas por uma equipe multidisciplinar junto aos idosos matriculados na disciplina osteoporose. As aulas ministradas para cada turma compreenderam um total de vinte encontros de duas horas por semana, perfazendo uma carga horária total de quarenta horas teóricas e práticas. Foram usados diversos recursos audiovisuais, como transparência, slides, material ilustrativo, músicas, dinâmica de grupo entre outros.

Nas atividades práticas foram feitos exercícios físicos sob orientação do educador físico e preparações culinárias enfatizando-se os alimentos fontes de cálcio, como leite e derivados, a sardinha e a soja, com supervisão e orientação de nutricionistas e monitores (estudantes de nutrição). Com o leite, preparou-se o iogurte, queijo, arroz de leite e com a soja foram feitos hambúrguer, pudim, bife e cuscuz em mistura com a sardinha. No final de cada aula prática era feita uma exposição das preparações e, uma vez saboreadas por todos, discutia-se a importância nutricional de cada uma delas.

Ao término do curso foi apresentada uma cartilha sobre osteoporose elaborada pelos participantes. Os trabalhos foram encerrados com uma confraternização, em que lanches ricos em cálcio e vitamina $\mathrm{D}$ eram trazidos por cada um para degustação, numa verdadeira 
demonstração de solidariedade e companheirismo.

A coleta dos dados foi realizada por acadêmicas de Nutrição previamente treinadas para aplicação dos questionários, supervisionadas sob a coordenação de um professor/pesquisador.

Os dados recolhidos foram analisados mediante a utilização do programa Epi Info 6.04B para as questões de múltiplas escolhas. $\mathrm{O}$ tratamento analítico das concepções foi feito a partir da leitura de cada uma das respostas obtidas, as quais foram organizadas em categorias temáticas e classificadas em percentuais por ordem crescente de freqüência, com o propósito de orientar as discussões.

Os aspectos éticos foram devidamente considerados e condicionados aos requisitos contidos nas Diretrizes e Normas Regulamentadoras de Pesquisa Envolvendo Seres Humanos, do Conselho Nacional de Saúde, Resolução no 196/1996 14 e o estudo foi submetido à apreciação e aprovado pelo Comitê de Ética da UFPI (003636/01-78).

\section{Resultados e discussão}

O interesse pela disciplina osteoporose foi demonstrado por cerca de cem idosos, $95 \%$ foram mulheres e 5\% homens. A grande presença de participantes do sexo feminino mostra não só um maior interesse das mulheres nesse tipo de atividade, mas a importância também da freqüência da doença neste gênero, além da estrutura demográfica da população idosa, que apresenta maior número de mulheres. É muito comum nos grupos de Terceira Idade o número de mulheres superarem o de homens.

Efetivamente participaram até o final da disciplina 95 alunos, havendo uma perda de 5\%, devido à desistência dos alunos por motivos de saúde pessoal ou de familiares.

Observou-se que cerca de $62,5 \%$ dos inscritos tinham osteoporose (exame de densitometria óssea), enquanto o restante ou não sabia se era realmente osteoporótico (25\%) ou não era doente $(16,5 \%)$ porque nunca havia feito o exame, por desconhecimento ou por não ter condições financeiras. Mesmo entre os diagnosticados como doentes, foi mencionada a identificação tardia da doença, ou seja, o exame era realizado já no estado avançado. Considerando-se que para a prevenção há necessidade do diagnóstico precoce, este não sendo realizado ou acontecendo em momentos tardios, compromete muito acompanhar a evolução que o paciente apresenta e monitorar o efeito do tratamento.
Em relação ao conhecimento do grupo sobre a doença, verificou-se que $66,7 \%$ tinham pouco conhecimento a respeito da doença e dos benefícios advindos da alimentação e da atividade física. Na descrição do que era osteoporose, apenas $33,3 \%$ conceituavam mais precisamente o que poderia ser essa doença como "desmineralização dos ossos", "ossos fracos por falta de cálcio". Enquanto outros descreviam como sendo "uma doença grave" ou "doença da terceira idade". Para muitos, o entendimento da doença não estava claro e era comum confundirem osteoporose com artrite, observação também identificada no estudo de Satterfield et al. 8 , onde um terço das idosas americanas não sabiam fazer diferenças entre as duas patologias.

A respeito das causas presumíveis da doença, grande parte dos entrevistados $(62,5 \%)$ não tinha idéia sobre o que poderia causar a doença e apenas $37,5 \%$ mencionaram a osteoporose como decorrência da "falta de cálcio e hormônio", "falta de cálcio, hormônio e exercício físico", mostrando que a alimentação e atividade física eram concepções bem valorizadas.

Sobre a sintomatologia da doença o que mais foi descrito pelos idosos foram as "dores nas articulações, membros e corpo".

A maioria dos entrevistados (62,5\%) procurou o serviço de saúde a partir do aparecimento dos sintomas, como as dores nas articulações, sendo que $25 \%$ apenas iniciaram o tratamento depois da ocorrência do estado mais grave.

Indivíduos com osteoporose apresentam riscos aumentados de fraturas 15 , no entanto, apenas uma das entrevistadas referiu uma queda com fratura no colo do fêmur. As quedas em idosos geralmente levam ao medo de cair, à restrição de atividades, ao declínio na saúde e ao aumento do risco de institucionalização, causando não só o prejuízo físico, mas psicológico e aumento dos custos com os cuidados de saúde 16. Mais de dois terços dos idosos que caem cairão novamente nos seis meses seguintes ao primeiro episódio, e, daqueles que necessitam de hospitalização, $15 \%$ a $20 \%$ morrem no primeiro ano 17. Apesar de saber que o comportamento do idoso frente ao risco, percebido durante a execução das atividades do cotidiano, aumenta a chance de cair, poucos tiveram atenção e cuidado com o ambiente dentro de casa.

Quanto aos tipos de tratamentos mais usados, foi mencionado o uso de hormônios e de suplementos de cálcio. Constatou-se que a maioria $(62,5 \%)$ dos idosos entrevistados estava consciente da necessidade do tratamento, enquanto $16,7 \%$ abandonaram o tratamento porque "estava muito caro"; e os que não o seguiam 
regularmente era por "displicência própria" ou "estava engordando". Além disso, vários entrevistados reclamaram dos efeitos colaterais atribuídos aos hormônios usados no tratamento da osteoporose e da menopausa. Uma das mulheres, por exemplo, relatou inchaço, sangramento e ganho de peso.

A respeito da ingestão de suplementos de cálcio, alguns idosos estavam conscientes da sua necessidade; e apenas $45,8 \%$ dos entrevistados usavam suplementos por orientação médica e/ou por conta própria. O suplemento mais usado foi Calcium 1000, na quantidade de um comprimido por dia. Um dos fatores que dificulta a aderência dos usuários ao tratamento é a dificuldade financeira, freqüentemente mencionada, para a obtenção do medicamento. Em Teresina, o medicamento da osteoporose Calcitonina Salmão Spray (Miacalcic nasal) passou a ser custeado pelo Governo Estadual somente a partir do dia 4 de abril de 2002 (informações fornecidas pela enfermeira responsável pela distribuição de medicamentos especiais).

A obtenção do medicamento pelos usuários não é muito fácil, observa-se uma demora no atendimento, burocracia excessiva, falta/atraso do medicamento na farmácia, desinformação, pouca divulgação e orientação aos usuários em relação ao protocolo para à aquisição do remédio e da existência deste serviço, dificultando a prevenção e a aderência à medicação para o tratamento da doença. Um total de dez pessoas está matriculado no programa de osteoporose para a obtenção do medicamento até 24 de março de 2003 e não há disponibilidade do Miocalcic na farmácia, diz a enfermeira. O medicamento só poderá ser disponibilizado aos pacientes cadastrados após o parecer favorável do auditor revisor da Secretaria de Saúde do Estado.

Quando abordados sobre a relação dieta e osteoporose, $70,8 \%$ desconheciam esta informação e $29,2 \%$ estavam conscientes porque receberam orientação médica e citaram "comer alimentos ricos em cálcio, sardinha, queijo, ovos", ou "tomar leite".

Dos indivíduos que mencionaram receber informação a respeito da doença, $66,7 \%$ foram repassadas pelo médico, 20,8\% pelos meios de comunicação e $12,5 \%$ por meio de revistas ou folhetos. Entre os que receberam informações sobre a alimentação $(n=9)$, apenas dois casos relataram que estas foram fornecidas por nutricionistas, o restante foi dado pelo médico. É necessária uma participação maior do nutricionista neste processo uma vez que os hábitos alimentares são importantes determinantes do estado de saúde dos indivíduos. A importância que assumiu a nutrição na prevenção, recuperação, tratamento e até na cura de determinadas doenças é amplamente descrita na literatura.

Observa-se não haver uma inserção do nutricionista em muitos serviços de saúde de Teresina, apesar da oferta desse profissional no mercado de trabalho. Seria interessante que houvesse um maior aproveitamento deste profissional, considerando que poderia prestar um apoio técnico e contribuir para o trabalho de prevenção. Isto reduziria os custos uma vez que o nutricionista recebe, na sua formação acadêmica, um forte estímulo para atuação em ações de Educação em Saúde.

$\mathrm{Na}$ análise das questões de consumo alimentar, os indivíduos (66\%) revelaram consumir café diariamente. No entanto, o hábito de consumo diário de leite e derivados integrais foi declarado apenas por $52 \%$, e desnatados, $42 \%$. O consumo de verduras e frutas, apesar de serem considerados alimentos saudáveis, apresentou-se baixo. Parcela importante de indivíduos $(72 \%)$, referiu à prática de consumir Coca-Cola pelo menos uma vez na semana.

Analisando-se os conhecimentos adquiridos após a aplicação das estratégias pedagógicas utilizadas observou-se que ao serem novamente entrevistados para conceituar osteoporose foram predominantes as afirmações: "diminuição da massa óssea causando enfraquecimento do osso"; "perda da massa óssea por falta de cálcio, principalmente nas mulheres na época da menopausa, se não existir reposição hormonal"; "doença sistêmica complexa caracterizada pela redução da massa óssea e conseqüente aumento de risco de fraturas"; "doença considerada silenciosa, assintomática”.

As principais causas da osteoporose foram apontadas como sendo a falta de cálcio, de hormônio e de exercício físico, como também o consumo excessivo de café, bebidas alcoólicas e o tabagismo.

Sobre os sintomas da doença, os idosos citavam principalmente: "dor no corpo, cansaço e fraqueza".

A dieta passou a ser muito valorizada após a intervenção e, quase todos passaram a consumir mais alimentos fontes de cálcio além da atenção aos outros constituintes alimentares na composição de uma dieta saudável. As expressões: "a dieta evita o progresso da doença"; "é importante uma boa alimentação, rica em cálcio"; "importante saber evitar alimentos que nos prejudiquem”, como excesso de sal, proteínas, gorduras, açúcares; "o cálcio ajuda no tratamento e prevenção da osteoporose"; "cálcio ajuda a fortificar os ossos"; "uso do hormônio com dieta rica em cálcio é importante para tra- 
tar e prevenir osteoporose: alimentos bons para osteoporose são leite, queijo, coalhada, iogurte, sardinha, soja, peixes, frutas e verduras".

Cerca de $81,8 \%$ dos idosos afirmaram ter feito alterações nos seus hábitos alimentares após a disciplina, apresentando como principais justificativas: "aprendemos a comer alimentos bons"; "os alimentos fontes de cálcio são importantes para prevenir a osteoporose"; "estava me alimentando de maneira errada"; "tive o conhecimento dos alimentos apropriados para o idoso"; "passei a tomar leite com vitamina D"; "consumir menos gordura, sal, carne vermelha e mais carne branca”.

Para os entrevistados, também foram considerados elementos de aprendizagem: "evitar a osteoporose"; "cuidar mais de mim e orientar os outros"; "alimentar-se melhor"; "ter cuidado para não cair"; "aprender a cuidar melhor da saúde e transmitir para as outras pessoas”; “é importante prevenir as quedas, que são responsáveis pelas fraturas e os perigos dentro de casa devem ser reduzidos ou eliminados".

Em relação à atividade física, todos foram encorajados a praticar e a maioria $(90 \%)$ passou a adotar essa prática, regularmente, principalmente, as atividades como a hidroginástica e a caminhada. Os benefícios do exercício cuidadoso e bem orientado são indiscutíveis e os participantes passaram a perceber a necessidade de realizar atividade física com as palestras e começaram a praticá-los. De acordo com Vuori 18 , a falta de atividade física e a nutrição inadequada agem sinérgica e conjuntamente na gênese de diversas doenças como Doenças Coronarianas e Cerebrovasculares, Hipertensão Arterial, Diabetes, Osteoporose e Obesidade. Conclui o autor que tanto a atividade física quanto à nutrição saudável devem ser enfatizados em quaisquer políticas, estratégias e programas de promoção da saúde.

Os dados de educação em osteoporose em mulheres na pré-menopausa 19,20 ou pós-menopausa 9 indicam que após a intervenção educativa essa estratégia pode contribuir para mudanças no estilo de vida, como redução no uso de cigarros, consumo de álcool e de bebidas cafeinadas. O uso de suplementos de cálcio e de vitamina $\mathrm{D}$ passou a ser mais valorizado, assim como o aumento na ingestão diária de leite.

Um programa de educação em osteoporose, sem dúvida, aumenta o nível de conhecimento, atenção e cuidados com a saúde óssea, mostrando a necessidade de um contínuo esforço em programas dessa natureza 21.

A educação, portanto, é um dos meios para vencer os desafios impostos aos idosos pela idade e pela sociedade, propiciando-lhes o apren- dizado de novos conhecimentos e oportunidades para buscar seu bem-estar físico e emocional. Os programas educacionais para idosos vêm procurando atender a essas necessidades, trabalhando com diversos procedimentos pedagógicos, a fim de despertar a consciência crítica para a busca de um envelhecer melhor 22 .

Na concepção de promoção da saúde, a aquisição de informações sobre os determinantes de saúde e doença desencadeia um processo no qual os indivíduos, agora tornados sujeitos graças à conscientização de sua situação de ser no mundo, buscam a sua autonomia para o enfrentamento dos seus problemas em direção a vidas mais saudáveis. A esse processo dá-se o nome de empoderamento (empowerment).

Esse processo ocorre não somente através de estratégias formais de ensino/aprendizagem, mas em todos os espaços sociais de convivência. Um desses espaços importantes atualmente são representados por experiências que ocorrem em universidades que promovem cursos, encontros, grupos de apoio proporcionando momentos importantes de interação entre os indivíduos. É fundamental trabalhar com os idosos em um ambiente acolhedor e facilitador, devendo-se incentivá-los a desenvolver novas capacidades, procurando desenvolver neles o autocuidado, para que possam adquirir comportamentos que conduzam à saúde.

O valor e o significado dessa intervenção educativa se justifica não somente pelos resultados positivos vivenciados pelos idosos, mas também pela eficácia constatada em outras pesquisas. O projeto de promoção da saúde da Universidade Aberta da Terceira Idade da Universidade do Estado do Rio de Janeiro (UNATI/ UERJ) reforça essas experiências uma vez que seus alunos tanto afirmam verbalmente quanto se esforçam por demonstrar em suas atitudes entusiásticas o nexo causal entre participar das atividades da UNATI/UERJ e poder experimentar um "envelhecimento adequado" 23.

Tendo consciência de que essa faixa etária possui características que lhes são peculiares, há necessidade de uma abordagem mais humanística com habilidades e dinâmicas que motivem o trabalho educacional com eles. É importante criar um clima psicológico que promova um ambiente encorajador e positivo, oferecendo subsídios para que todos os participantes possam lutar por uma alimentação mais saudável e, também, discutindo formas de obter condições de vida mais dignas.

Na avaliação do curso, 81,8\% dos entrevistados informaram ter achado ótimo e o restante relatou ter achado bom. A maioria $(77,2 \%)$ dos entrevistados afirmou ter sido ótimo a pro- 
gramação do curso. Todos afirmaram que as palestras não eram cansativas, foram bem explicadas e interessantes, e que o tempo se tornava curto.

Sobre o entendimento das palestras, $68,3 \%$ dos idosos afirmaram tê-las entendidos bem, enquanto $31,8 \%$ relataram ter entendido apenas algumas palestras. Foram comuns às afirmações: "todas as aulas foram boas"; "deu para entender"; "tivemos bom aproveitamento"; "quando se vê, se aprende melhor"; "tudo foi muito bem explicado e transmitido com esclarecimento, dedicação e paciência"; "estava tudo ótimo e muito bom".

Dentre os temas que mais tiveram dificuldades de entendimento foram citados: "o que é osteoporose"; "fatores que interferem na absorção do cálcio", principalmente quando se falava "nome científico" ou porque "tinham dificuldade de memória".

Os temas preferidos por $86,3 \%$ dos entrevistados foram os que abordavam a doença, alimentação e nutrição. Apenas 13,6\% mencionaram os temas ligados a atividade física.

Foi consenso entre todos a importância da disciplina para o idoso. Cerca de $68,1 \%$ enfatizaram a necessidade de expandir o curso para outros grupos e quase todos passaram a valorizar mais a dieta e a atividade física, adotando atitudes mais corretas no tratamento. Mostraram-se mais conscientes e relataram que aprenderam muito sobre a doença, prevenção e tratamento. A maioria referiu: "aprendemos muito, tivemos bom proveito"; "é bom que este curso não termine para que o idoso tenha oportunidade de aprender, para saber e se cuidar melhor"; "acho que deveria ser um período mais longo, com mais tempo para falar sobre esta doença, osteoporose...”; "queria que o curso durasse mais tempo".

Em relação aos fatores de risco para osteoporose presentes no grupo estudado e que foram desaparecendo com as ações educativas destacam-se a inclusão de práticas de atividade física, a mudança nos hábitos alimentares como o maior consumo de leite e derivados, alimentos enriquecidos, soja e espinafre; mais interesse e cuidado no ato de alimentar-se, no preparo das refeições e seleção de alimentos para consumo; a redução no consumo de café, refrigerantes, carnes e salgados.

A reação positiva dos idosos frente às atividades programadas no curso pode ser devido ao aprendizado, o vínculo afetivo com o grupo, o estímulo/motivação para a vida e o impacto na saúde. Uma das manifestações mais comuns é o lamento pelo término do trabalho e o desejo de continuar.

\section{Considerações finais}

Os dados obtidos mostraram um grande desconhecimento da população entrevistada a respeito da osteoporose e dos cuidados que se deve ter para melhorar e controlar a progressão da perda da massa óssea. A pouca informação sobre a doença mostra a dificuldade na prática médica de se estabelecer uma melhor forma de controle e tratamento. Após o curso, pudemos constatar que os idosos estão bem mais informados a respeito da necessidade e dos cuidados no tratamento da osteoporose, passaram a enfrentar a doença de forma mais adequada e adotaram hábitos de vida mais saudáveis, principalmente, em relação à dieta e à atividade física.

\section{Resumo}

O objetivo do estudo foi análise do conhecimento, concepções e mudanças de comportamento alimentar de idosos universitários em relação à temática osteoporose, antes e após a intervenção educativa. Utilizou-se a estratégia da pesquisa-ação, aplicando-se o questionário a 95 idosos, com idade entre 60 e 86 anos, com ou sem osteoporose. O trabalho tornou patente o desconhecimento dos idosos em informações importantes sobre a doença e dos cuidados que se deve ter para melhor controlar a progressão da perda da massa óssea. Após o período de quatro meses de atividades educati- vas mais da metade do grupo reportou mudanças na dieta. Enfatiza-se a necessidade de adotar estratégias educativas capazes de informar não só sobre as práticas preventivas ideais da osteoporose, mas também que possam construir uma nova mentalidade e um novo comportamento que sejam importantes para o controle da doença.

Osteoporose; Envelhecimento; Educação em Saúde; Hábitos Alimentares; Promoção da Saúde 


\section{Colaboradores}

C. M. R. G. Carvalho colaborou na discussão dos resultados, redação completa do artigo e revisão da prova de prelo. C. C. C. Fonseca contribuiu com o levantamento bibliográfico, aplicação de questionários e digitação dos dados. J. J. Pedrosa colaborou na leitura e sugestões na revisão do texto.

\section{Referências}

1. Chau DL, Edelman SV. Osteoporosis and diabetes. Clin Diabetes 2002; 20:153-7.

2. Nightingale S, Holmes J, Mason J, House A. Psychiatric illness and mortality after hip fracture. Lancet 2001; 357:1264.

3. Ray NF, Chan JK, Thamer M, Melton LJ. Medical expenditures for the treatment of osteoporotic fracture in the United States in 1995: report from the National Osteoporosis Foundation. J Bone Miner Res 1997; 12:24-35.

4. Kida AA, Takimoto L, Mondini L, Freire DR, Lai DLM. Osteoporose e a saúde da mulher. Mundo Saúde 1999; 23:120-4.

5. Carneiro RA. Osteoporose: problema econômico a social de mundial. Osteotopics - Tópicos em Osteoporose 1998; 4:6-9.

6. Ministério da Saúde. Política nacional de promoção da saúde (documento para discussão), Brasília: Ministério da Saúde; 2002.

7. Sedlak CA, Doheny MO, Jones SL. Osteoporosis education programs: changing knowledge and behaviors. Public Health Nurs 2000; 17:398-402.

8. Satterfield T, Johnson SM, Slovic P, Neil N, Schein JR. Perceived risks and reported behaviors associated with osteoporosis and its treatment. Women Health 2000; 31:21-40.

9. Roinick SJ, Kopher R, Jakcon J, Fischer LR, Compo R. What is the impact of osteoporosis education and bone mineral density testing for postmenopausal women in a managed care setting? Menopause 2000; 8:141-8.

10. Veras RP. País jovem com cabelos brancos: a saúde do idoso no Brasil. Rio de Janeiro: Relumé-Dumará/Universidade Aberta da Terceira Idade, Universidade do Estado do Rio de Janeiro; 1994.

11. Plaper P. Escola ajuda a reabilitar osteoporóticos. Osteotopics - Tópicos em Osteoporose 1998; 4:102 .

12. Berquó E. Considerações sobre o envelhecimento da população no Brasil. In: Neri AL, Debert GG, organizadores. Velhice e sociedade. Campinas: Papirus; 1999. p. 11-40.

13. Thiollent M. Metodologia da pesquisa-ação. 2a Ed. São Paulo: Editora Cortez; 1998.

\section{Agradecimentos}

Nossos agradecimentos e parabéns a Dra. Aglair Alencar Setúbal pela criação desta preciosa obra: o Programa Terceira Idade em Ação (PTIA). Também agradecemos aos idosos do PTIA pela participação valiosa e ativa; e destacamos a importante colaboração das estagiárias de nutrição: Celma de Oliveira Barbosa, Karilene Cardoso da Silva, Floriza Rodrigues de Rezende Monte, Maria do Socorro Nunes Sousa e Heulenmacya Rodrigues Matos. Agradecemos também ao médico reumatologista, médico ginecologista, educador físico, fisiologista e psicóloga pelas palestras ministradas. Este projeto foi desenvolvido com o apoio do Programa de Iniciação Científica (PIBIC/CNPq).

14. Ministério da Saúde. Diretrizes e normas regulamentadoras de pesquisas envolvendo seres humanos. Conselho Nacional de Saúde, Resolução 196/96. Brasília: Ministério da Saúde; 2000.

15. O'Brien KO. Combined calcium and vitamin D supplementation reduces bone loss and fracture incidence in older men and women. Nutr Rev 1998; 56:148-58.

16. Perracini MR, Ramos LR. Fatores associados a quedas em uma coorte de idosos residentes na comunidade. Rev Saúde Pública 2002; 36:1-15.

17. Baraff LJ, Della Penna R, Williams N, Sanders A. Practice guideline for the ED management of falls in community-dwelling elderly persons. Ann Emerg Med 1997; 30:480-92.

18. Vuori IM. Health benefits of physical activity with special reference to interaction with diet. Public Health Nutr 2001; 4:17-28.

19. Jamal AS, Ridout R, Chase C, Fielding L, Rubin LA, Hawker GA. Bone mineral density testing and osteoporosis education improve lifestyle behaviors in premenopausal womem: a prospective study. J Bone Miner Res 1999; 14:2143-9.

20. Piaseu N, Belza B, Mitchell P. Testing the effectiveness of an osteoporosis educational program for nursing students in Thailand. Arthritis Rheum 2001; 45:246-51.

21. Berarducci A, Lengacher CA, Keller R. The impact of osteoporosis continuing education on nurses knowledge and attitudes. J Contin Educ Nurs 2002; 33:210-6.

22. Neri AL, Cachioni M. Velhice bem-sucedida e educação. In: Neri AL, Debert GG, organizadores. Velhice e sociedade. Campinas: Papirus; 1999. p. 113-40.

23. Lima MA. A gestão da experiência de envelhecer em um programa para a terceira idade: a UNATI/ UERJ. Textos sobre Envelhecimento 1999; 2:33-99.

Recebido em 25/Nov/2002

Versão final reapresentada em 27/Jun/2003 Aprovado em 23/Out/2003 